OPEN ACCESS

Edited by:

Matteo Lambertini,

Università degli Studi di Genova, Italy

Reviewed by:

Francesco Schettini,

Institut de Recerca Biomèdica August

Pi i Sunyer (IDIBAPS), Spain

Gaia Griguolo,

Università di Padova, Italy

*Correspondence:

Jai Min Ryu

jaimin.ryu@samsung.com

Specialty section:

This article was submitted to

Breast Cancer,

a section of the journal

Frontiers in Oncology

Received: 06 April 2021 Accepted: 07 June 2021

Published: 21 June 2021

Citation:

Lee J-H, Lee SK, Chae BJ, Yu J, Lee JE, Kim SW, Nam SJ and Ryu JM

(2021) Validation of the Clinical Treatment Score Post-Five Years in Breast Cancer Patients for Predicting Late Distant Recurrence: A SingleCenter Investigation in Korea.

Front. Oncol. 11:691277. doi: 10.3389/fonc.2021.691277

\section{Validation of the Clinical Treatment Score Post-Five Years in Breast Cancer Patients for Predicting Late Distant Recurrence: A Single-Center Investigation in Korea}

\author{
Jun-Hee Lee, Se Kyung Lee, Byung Joo Chae, Jonghan Yu, Jeong Eon Lee, \\ Seok Won Kim, Seok Jin Nam and Jai Min Ryu*
}

Division of Breast Surgery, Department of Surgery, Samsung Medical Center, Sungkyunkwan University School of Medicine, Seoul, South Korea

Background: Endocrine therapy is administered to hormone-positive breast cancer patients to prevent distant metastasis. It is important to evaluate the risk of recurrence and to determine which patients are viable candidates for such treatment because hormone therapy has side effects that can include postmenopausal symptoms. The Clinical Treatment Score post-five years (CTS5), a simple tool for identifying candidates for endocrine therapy, was recently introduced; however, CTS5 only has been applied in validation studies with postmenopausal women. We aimed to validate CTS5 among premenopausal breast cancer patients.

Methods: We identified patients treated between 1994 and 2014 at Samsung Medical Center in Seoul, Korea, and followed their treatment outcomes for more than 60 months after surgery using clinicopathologic parameters. According to menopausal status, we divided the study population into two groups: pre- and postmenopausal women. After calculating CTS5 values based on some parameters, we stratified the rate of late distant recurrence (DR) and analyzed the correlation between CTS5 value and late DR by risk.

Results: Among 16,904 patients treated surgically for breast cancer, 2,605 with hormone receptor-positive breast cancer who received endocrine therapy were included. Of these, $1,749(67.14 \%)$ patients were premenopausal women, and the median age was 44.00 years. When categorizing study participants according to CTS5-related risk for late DR, 86.79\% were categorized as low risk, 5.95\% were categorized as intermediate risk, and $7.26 \%$ were categorized as high risk. The annual rate of DR was $1.41 \%$ for those in the present study and was similar between pre- and postmenopausal participants (1.40 vs. 1.42). Distant metastasis-free survival was not different between the two groups (hazard ratio: $0.817,95 \%$ confidence interval $[\mathrm{Cl}]$ : 0.547-1.221). The area under the receiver operating characteristic curve at 10 years for premenopausal and postmenopausal patients was 61.75 (95\% Cl: 52.97-70.53) and 72.71 (95\% Cls: 63.30-82.12), respectively. 
Conclusions: Although CTS5 was able to predict late DR, it should be applied with caution in premenopausal women. A CTS5 calculator for premenopausal women might be needed to not underestimate the risk of recurrence in Korea.

Keywords: hormone receptor-positive breast cancer, premenopausal patients, hormone replacement therapy, CTS5, late distant recurrence

\section{INTRODUCTION}

Endocrine therapy is inevitable for patients with hormonal status-positive breast cancer to prevent local recurrence and distant metastasis (1-3). Generally, patients with estrogen receptor (ER)- or progesterone receptor (PR)-positive breast cancer are treated with adjuvant endocrine therapy for five years after surgical treatment $(4,5)$. It is important to evaluate the recurrence risk and determine whether to maintain or stop endocrine therapy after five years based on side effects, such as postmenopausal symptoms, and patient quality of life (6-8). Therefore, it is necessary to decide whether to stop or continue endocrine therapy after weighing the side effects of therapy and the risk for recurrence or metastasis of breast cancer.

Recently, Dowsett and colleagues introduced a tool called the Clinical Treatment Score post-five years (CTS5) as a scoring system to help decide whether to stop or continue treatment after five years of endocrine therapy using several clinicopathologic parameters including tumor size, nodal status, and histopathologic grade (9, 10). This scoring system was developed using data from the Arimidex, Tamoxifen, Alone or in Combination (ATAC) trial, which included postmenopausal women with ER-positive or ERunknown early breast cancer $(11,12)$. The ATAC trial categorized patients into three risk groups (low, intermediate, and high) for estimating the prognostic performance for late distant metastasis.

However, the CTS5 scoring system might not be as effective in Asian countries because there are many more young breast cancer patients than in Western society (13). In prior research, CTS5 was applied to postmenopausal women in the ATAC and BIG 1-98 study cohorts at diagnosis, and the algorithm was not applied to premenopausal patients $(11,14,15)$. CTS5 provides a convenient way to predict distant recurrence (DR) but has limitations in extending its use to all ER- or PR-positive breast cancer patients.

In the present study, we aimed to validate the CTS5 score and develop a modified scoring system to predict distant metastasis not only in postmenopausal women, but also in premenopausal women. We used data from a single institution as the validation set and analyzed participants after subdividing them into preand postmenopausal groups to differentiate existing CTS5 scores and identify the prognostic value of CTS5 according to menopausal status.

\section{PATIENTS AND METHODS}

\section{Study Populations}

We retrospectively reviewed the medical records of patients who were treated surgically for breast cancer at Samsung Medical
Center in Seoul, Korea, between January 1994 and December 2014. Among them, patients with hormone receptor-positive early breast cancer who received adjuvant endocrine therapy and were followed for more than 60 months after surgery were included. We excluded data from women with a final pathologic stage equal to or higher than T3 or N3, ductal carcinoma in situ, or a diagnosis of bilateral breast cancer. Patients who received neoadjuvant chemotherapy also were excluded. Patients with poor drug compliance-those with discontinuation of tamoxifen or aromatase inhibitors such as anastrozole or letrozole intake after starting - also were excluded. Additionally, patients who showed DR prior to five years after diagnosis were excluded from the study cohort. Finally, we excluded patients with extension of adjuvant endocrine therapy after five years (Figure 1). According to menstrual cycle period, date of last menstruation, and hormonal test results including follicle-stimulating hormone and estradiol levels, we divided the study population into two groups of pre- and postmenopausal women. Human epidermal growth factor receptor 2 (HER2)-positive patients were included.

\section{Validation as a Prognostic Tool}

Dowsett et al. (9) suggested the use of CTS5 for predicting late DR rates after five years of adjuvant endocrine therapy in patients with hormonal receptor-positive breast cancer. Using the formula CTS5 $=0.438 \times$ nodes $+0.988 \times(0.093 \times$ size $0.001 \times$ size $^{2}+0.375 \times$ grade $+0.017 \times$ age $)$, we validated CTS5 as a prognostic tool for $\mathrm{DR}$ onset. We assigned three risk categories in each group of women according to cutoff values of $5 \%$ and $10 \%$ of DR risk as calculated by CTS5. The cutoff criteria for classifying risk were the same as those of CTS5 values for the combined dataset (ATAC training set and BIG 1-98 validation set).

For survival analysis, the five- to 10 -year DR risk was analyzed for each group by Kaplan-Meier plots. The hazard ratio (HR) and 95\% confidence interval (CI) of the premenopausal group were calculated and compared to those of the postmenopausal group through univariate analysis. Timedependent areas under the receiver operating characteristic curve (AUC) at 10 years with 95\% CIs were calculated to evaluate matching of the DR rate prediction in between pre- and postmenopausal groups.

\section{Statistical Analyses}

Patient characteristics were compared using the independent $t$ test for continuous variables and the chi-square or Fisher's exact test for categorical variables. Univariable and multivariable analyses were conducted using Cox regression analysis models. 


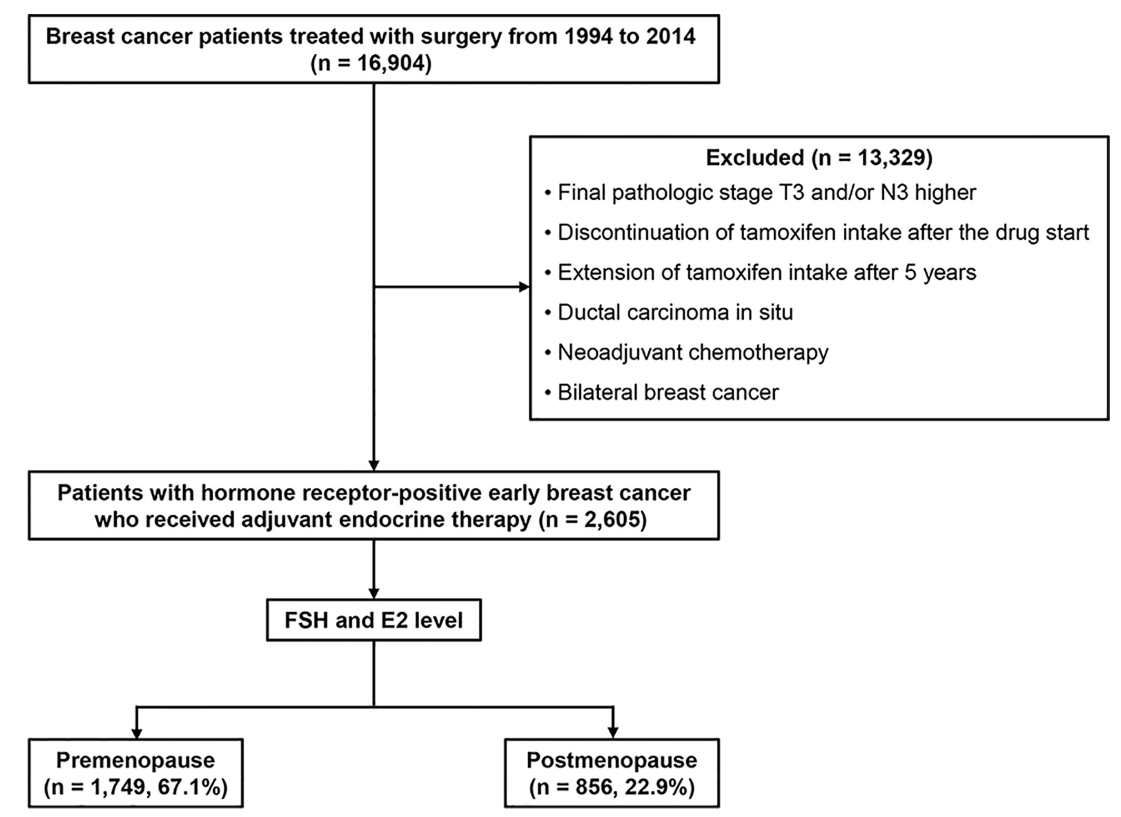

FIGURE 1 | Consort diagram of the study population.

The five- to 10-year DR risk was estimated using the KaplanMeier method. Kaplan-Meier curves, with corresponding logrank tests, were constructed for DR. Values are reported as mean \pm standard deviation (SD) or median with range.

Statistical significance was established at $\mathrm{p}<0.05$. All statistical analyses were performed using the Statistical Analysis System (SAS) version 9.4 (SAS Institute Inc., Cary, NC, USA) and R Statistical Programming Language Version 2.13.2 (The R Foundation, Vienna, Austria; available at http:// www.R-project.org/). The predictability of each CTS5 model for 10 -year distant metastasis-free survival was assessed with the time-dependent area under the curve (AUC) and its 95\% CI by constructing the receiver operating characteristic (ROC) curve at 10 years post-surgery using $\mathrm{R}$ package (16). The present study was approved by the Review Committees (no. 2020-09-143), and work was conducted according to the principles outlined in the Declaration of Helsinki.

\section{RESULTS}

The demographics and baseline characteristics of this study are described in Table 1. Among 2,605 patients included in the present study, $1,749(67.14 \%)$ were premenopausal, and 856 (32.86\%) were postmenopausal. The median follow-up period was 94.69 months [59.97 - 233.85]. The median age of the premenopausal women was 44.00 years, and that of the postmenopausal women was 56.50 years; overall, the average median age of the study population was 46.00 years. Nodal status, tumor grade, and tumor size were not significantly different between the two groups. A total of 1,902 patients
(73.04\%) received adjuvant chemotherapy, and more premenopausal women than postmenopausal women received chemotherapy $(77.02 \%$ vs. $64.91 \%$; $<<0.0001)$. There was no difference between pre- and postmenopausal patients in terms of tumor size, tumor grade, or nodal status. Tamoxifen was administered in 1,481 patients $(56.85 \%)$ total, 1,325 of whom were premenopausal. Goserelin treatment as a subcutaneous injection of a depot formulation was observed in 159 patients, all of whom were premenopausal. As for aromatase inhibitors, 336 postmenopausal patients $(39.25 \%)$ received anastrozole, and 278 (32.48\%) received letrozole. During the first five years of treatment, 126 patients were switched to aromatase inhibitors, and all 126 patients were classified as premenopausal. Moreover, $110(4.22 \%)$ cases of late DR were recorded, with an annual hazard rate of $1.41 \%$ (95\% CI: $1.16 \%-1.70 \%)$. There was no significant difference between the two groups in terms of DR cases or annual rate of DR after five years of adjuvant endocrine therapy. The rate for late DR of HER2-positive patients was significantly lower than that of HER2-negative patients $(1.64 \%$ vs. 4.49\%; $\mathrm{p}=0.0351$ ) (Supplementary Table 1).

Tumor size, tumor grade, and nodal status were arranged in a separate table according to CTS5 risk category status in pre- and postmenopausal women (Table 2). Overall, 86.79\% ( $\mathrm{n}=2,261$ patients) were categorized as low risk, $5.95 \%$ ( $n=155$ patients) were categorized as intermediate risk, and 7.26\% ( $\mathrm{n}=189$ patients) were categorized as high risk for late DR. Notably, more than $90 \%$ of patients in the moderate to poor tumor grade group were categorized high risk, as were all patients with more than two positive nodes. Combined ATAC and BIG 1-98 cohort data and data from participants of this study are compared in Table 3. Compared with the ATAC and BIG 1-98 cohorts, there 
TABLE 1 | Demographic and clinical characteristics between pre- and postmenopausal women.

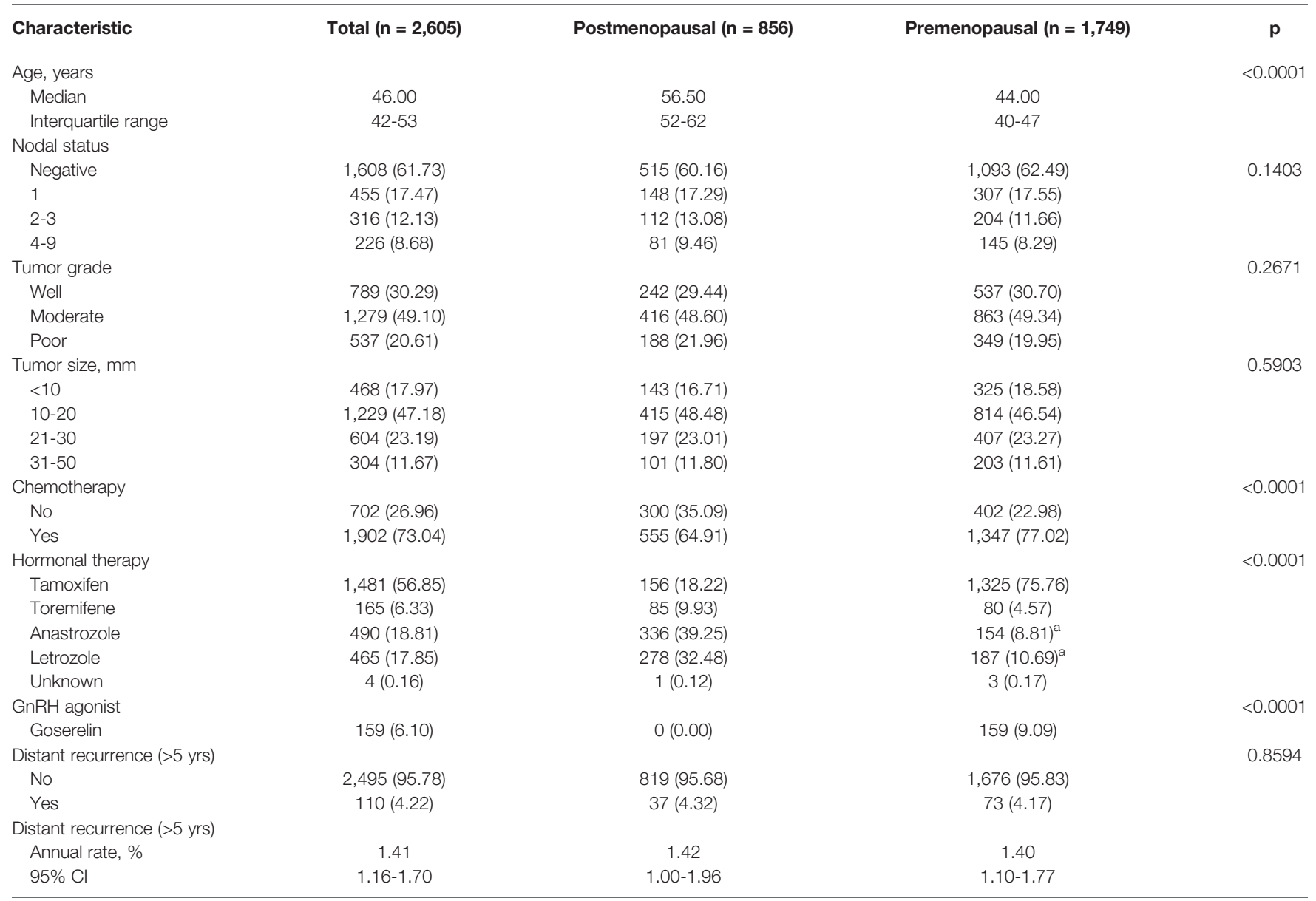

${ }_{126}$ patients were switched from tamoxifen to aromatase inhibitors during the first 5 years of treatment.

was no difference in tumor size or nodal status. The rate of chemotherapy in our study was more than $70 \%$ higher than that of the ATAC and BIG 1-98 cohorts, representing a significant difference, and low risk was identified in more than $80 \%$ of patients with the CTS5 score at the time of validation. The HR of five- to 10 -year DR risk among premenopausal women was 0.817 (95\% CI: $0.547-1.221 ; \mathrm{p}=0.3236$ ), which was lower than that among postmenopausal women (Figure 2).

The time-dependent AUC at 10 years is presented with $95 \%$ CI value (Figure 3). The AUC for all patients was 64.71 (95\% CI: 57.75-71.67). Among postmenopausal women, the AUC exceeded the total population AUC at 72.71 (95\% CI: $57.75-$ $71.67)$, whereas that in premenopausal women was $61.75(95 \%$ CI: 52.97-70.53).

Histograms for CTS5 score are shown according to menopausal status with validated prognostic values of CTS5 for risk of DR between five and 10 years (Figure 4). Importantly, the premenopausal group included a greater proportion of patients at low risk than did the postmenopausal group. That is, premenopausal women unexpectedly had lower CTS5 scores than postmenopausal women, and this result correlated with the many patients at low risk.

\section{DISCUSSION}

There is a need for prognostic tools that can predict late recurrence rate after five years of endocrine therapy; CTS5 is a useful tool for satisfying this need and supporting clinicians in decision-making regarding extension of endocrine therapy (9, 17). This study is significant in that CTS5 was validated in premenopausal women, who account for the majority of breast cancer patients in Korea. After validation, we found that premenopausal women occupied a large portion of the lowrisk recurrence group-in other words, the risk for late DR was underestimated by CTS5 in premenopausal women. Therefore, development of a predictive late DR model for premenopausal women is necessary.

Notably, compared with our study population, the ATAC cohort, used as the training set in CTS5, and the BIG 1-98 cohort, used as the validation set, both included postmenopausal women. Nodal status, tumor grade, and tumor size were similar between the population in our study and the ATAC plus BIG 1-98 cohort. In our study, there was no significant difference in tumor size, tumor grade, or nodal status between pre- and postmenopausal women. There was also no difference 
TABLE 2 | Distribution of risk categories according to menopausal status.

\begin{tabular}{|c|c|c|c|c|}
\hline Characteristic & Total $(n=2,605)$ & Low Risk ( $n=2,261)$ & Intermediate Risk (n = 155) & High Risk $(n=189)$ \\
\hline \multicolumn{5}{|c|}{ Postmenopausal ( $n=856$ ) } \\
\hline \multicolumn{5}{|l|}{ Tumor size, mm } \\
\hline$<10$ & $143(16.71)$ & $142(19.8)$ & $1(1.61)$ & $0(0)$ \\
\hline $10-20$ & $415(48.48)$ & $375(52.3)$ & $23(37.1)$ & $17(22.08)$ \\
\hline $21-30$ & $197(23.01)$ & $142(19.8)$ & 18 (29.03) & $37(48.05)$ \\
\hline $31-50$ & $101(11.8)$ & $58(8.09)$ & $20(32.26)$ & $23(29.87)$ \\
\hline \multicolumn{5}{|l|}{ Tumor grade } \\
\hline Well & $252(29.44)$ & 239 (33.33) & $8(12.9)$ & $5(6.49)$ \\
\hline Moderate & $416(48.6)$ & 351 (48.95) & $27(43.55)$ & 38 (49.35) \\
\hline Poor & $188(21.96)$ & $127(17.71)$ & $27(43.55)$ & $34(44.16)$ \\
\hline \multicolumn{5}{|l|}{ Nodal status } \\
\hline Negative & $515(60.16)$ & $515(71.83)$ & $0(0)$ & $0(0)$ \\
\hline 1 & $148(17.29)$ & $135(18.83)$ & $13(20.97)$ & $0(0)$ \\
\hline $2-3$ & $112(13.08)$ & $66(9.21)$ & $33(53.23)$ & $13(16.88)$ \\
\hline $4-9$ & $81(9.46)$ & $1(0.14)$ & $16(25.81)$ & $64(83.12)$ \\
\hline \multicolumn{5}{|c|}{ Premenopausal $(n=1,749)$} \\
\hline \multicolumn{5}{|l|}{ Tumor size, mm } \\
\hline$<10$ & $325(18.58)$ & $322(20.85)$ & $1(1.08)$ & $2(1.79)$ \\
\hline $10-20$ & $814(46.54)$ & $779(50.45)$ & $16(17.2)$ & 19 (16.96) \\
\hline $21-30$ & 407 (23.27) & $315(20.4)$ & $44(47.31)$ & $48(42.86)$ \\
\hline $31-50$ & $203(11.61)$ & $128(8.29)$ & $32(34.41)$ & $43(38.39)$ \\
\hline \multicolumn{5}{|l|}{ Tumor grade } \\
\hline Well & $537(30.7)$ & $523(33.87)$ & $5(5.38)$ & $9(8.04)$ \\
\hline Moderate & $863(49.34)$ & $751(48.64)$ & $55(59.14)$ & $57(50.89)$ \\
\hline Poor & 349 (19.95) & $270(17.49)$ & 33 (35.48) & $46(41.07)$ \\
\hline \multicolumn{5}{|l|}{ Nodal status } \\
\hline Negative & $1,093(62.49)$ & $1,093(70.79)$ & $0(0)$ & $0(0)$ \\
\hline 1 & 307 (17.55) & $302(19.56)$ & $5(5.38)$ & $0(0)$ \\
\hline $2-3$ & $204(11.66)$ & $140(9.07)$ & $58(62.37)$ & $6(5.36)$ \\
\hline $4-9$ & $145(8.29)$ & $9(0.58)$ & $30(32.26)$ & $106(94.64)$ \\
\hline
\end{tabular}

TABLE 3 | Comparison of combined ATAC and BIG 1-98 cohorts and the present cohort.

\begin{tabular}{|c|c|c|c|c|}
\hline Characteristic & ATAC + BIG 1-98 $(n=11,446)$ & Total $(n=2,605)$ & Premenopausal $(n=1,749)$ & $\mathbf{p}$ \\
\hline Nodal status & & & & 0.1519 \\
\hline Negative & $7,309(63.86)$ & $1,608(61.73)$ & $1,093(62.49)$ & \\
\hline 1 & $1,807(15.79)$ & $455(17.47)$ & $307(17.55)$ & \\
\hline $2-3$ & $1,303(11.38)$ & $316(12.13)$ & $204(11.66)$ & \\
\hline $4-9$ & 1,027 (8.97) & $226(8.68)$ & 145 (8.29) & \\
\hline Tumor grade & & & & $<0.0001$ \\
\hline Well & $2,673(23.35)$ & 789 (30.29) & $537(30.70)$ & \\
\hline Moderate & $6,215(54.30)$ & $1,279(49.10)$ & $863(49.34)$ & \\
\hline Poor & $2,558(22.35)$ & $537(20.61)$ & 349 (19.95) & \\
\hline Tumor size, mm & & & & 0.6291 \\
\hline$<10$ & 2,036 (17.79) & 468 (17.97) & $325(18.58)$ & \\
\hline $10-20$ & $5,562(48.59)$ & $1,229(47.18)$ & $814(46.54)$ & \\
\hline $21-30$ & $2,599(22.71)$ & $604(23.19)$ & 407 (23.27) & \\
\hline $31-50$ & $1,249(10.91)$ & $304(11.67)$ & $203(11.61)$ & \\
\hline Chemotherapy & & & & $<0.0001$ \\
\hline No & 8,896 (77.72) & 702 (26.96) & $402(22.98)$ & \\
\hline Yes & $2,550(22.28)$ & $1,902(73.04)$ & $1,347(77.02)$ & \\
\hline Distant recurrence (>5yrs) & & & & $<0.0001$ \\
\hline No & $10,746(93.88)$ & $2,495(95.78)$ & $1,676(95.83)$ & \\
\hline Yes & $700(6.12)$ & $110(4.22)$ & $73(4.17)$ & \\
\hline CTS5 & & & & $<0.0001$ \\
\hline Low & $4,850(42.37)$ & 2,261 (86.79) & $1,544(88.28)$ & \\
\hline Intermediate & 3,620 (31.63) & 155 (5.95) & 93 (5.32) & \\
\hline High & 2,976 (26.00) & $189(7.26)$ & $112(6.40)$ & \\
\hline
\end{tabular}




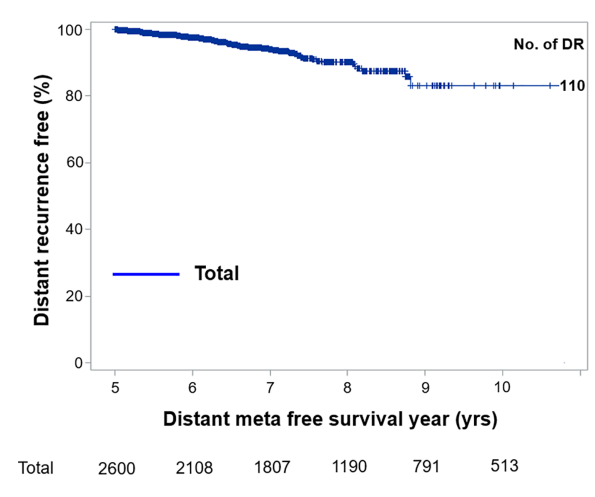

FIGURE 2 | Kaplan-Meier curves of distant recurrence-free survival.

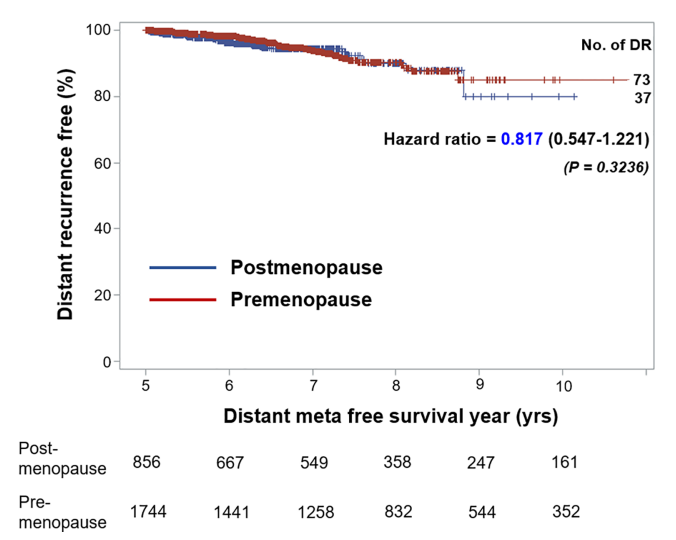

in DR or annual DR between the two groups. However, when risk was divided according to the cutoff value of CTS5, patients at low risk were more than twice as numerous relative to the cohorts of the ATAC and BIG 1-98 trials. In addition, the premenopausal group contained a greater number of patients at intermediate and high risk than did the postmenopausal group. Interestingly, the rate of receiving chemotherapy was close to $80 \%$ in our study, while about $20 \%$ of the ATAC plus BIG 1-98 cohort received adjuvant chemotherapy. For this reason, it is thought that the frequency of adjuvant chemotherapy was high among premenopausal women. The AUC had a greater predictive rate for postmenopausal women than for total patients but was less predictive for premenopausal women than for the entire cohort. In other words, as CTS5 was created based on data of postmenopausal women, there is a high probability that it cannot efficiently be applied to premenopausal women in many Asian countries (18). Premenopausal women are likely to be classified in an underestimated risk group when CTS5 is used, and it is necessary to introduce a new scoring system to address this.

Recently, many studies have analyzed the prognostic value of CTS5. Villasco et al. (19) found that CTS5 has prognostic value in predicting late DR in both pre- and postmenopausal women by testing its clinical validity in a retrospective cohort, while Lee et al. (20) similarly concluded that CTS5 is a good prognostic tool for evaluating the risk of late distant recurrence in both pre- and postmenopausal women using the Ki-67 labeling index and confirmed its prognostic performance in premenopausal women. Although Lee et al. reported that their risk groups presented differences in tumor grade relative to the ATAC and BIG 1-90 cohorts, there was no significant difference in tumor grade between our group and the ATAC plus BIG 1-90 cohort. We think that the reason for the different risk groups might be related to whether or not adjuvant chemotherapy was available.

HER2 gene amplification is known to have an impact on breast cancer, and the intracellular signaling pathway of estrogen receptors and HER2 has a complex connection (21). Wang et al. (22) recently suggested that HER2 status has an effect on late DR in hormone receptor-positive breast cancer. In HER2-positive patients, such as those with luminal type B disease, a less effective prognostic value was found in their study. Cases of HER2positive breast cancer are considered high-risk for DR but, due to the development of anti-HER2-targeted therapy, it is not effective for the HER2-positive model, and a further prediction model is required.

Recently, Noordhoek et al. (23) published a validity and accuracy study of the CTS5 for predicting the rate of late DR in the TEAM and IDEAL trials, arguing the CTS5 overestimates the risk of late DR in high-risk subgroups and suggesting that CTS5 should be used cautiously for interpreting the DR rate among patients at high risk despite its ease of use. Based on these findings, CTS5 must be applied carefully, and unique validation is justified in Korea, where there are many young breast cancer patients.

Various multigene assays have been developed to complement predictions using existing clinicopathologic parameters because of the lack of predictive values for late DR. Among them, GenesWell ${ }^{\mathrm{TM}}$ BCT has been validated as a tool to predict late DR (24). In some patients who underwent CTS5 validation, GenesWell ${ }^{\mathrm{TM}}$ BCT also was applied, and the CTS5derived risk group correlated with the gene assay risk group (Supplementary Figure 1). However, the use of multigene assays is controversial in predicting late DR because some assays have not been validated, and the importance of clinical risk has been emphasized, especially in multigene panels of patients under 50 years of age (25). In conclusion, balanced application of a clinicopathologic prediction model and a multigene assay should be conducted to predict late DR, and further study is needed for proper selection of patients to receive extended adjuvant endocrine therapy.

The main limitation of this study is that it was a retrospective study conducted in a single center. It is necessary to conduct multicenter studies to further explore the limitations of CTS5, and a modified version of CTS5 should be developed and validated in large sets through multicenter research in South Korea. In addition, it is necessary to accurately stratify risk 


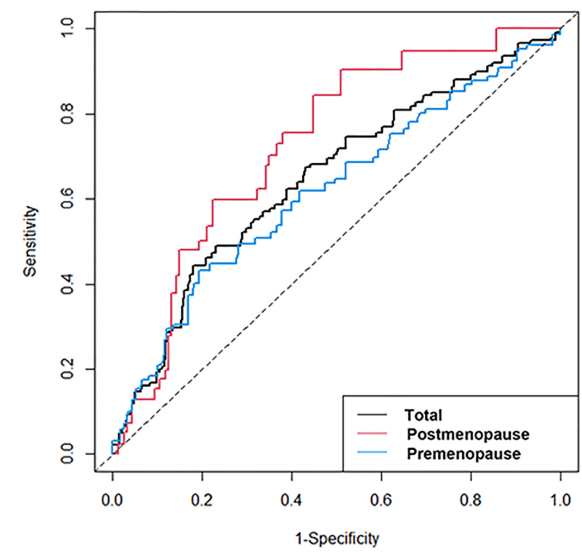

\begin{tabular}{cccc}
\hline & AUC & \multicolumn{2}{c}{$95 \% \mathrm{Cl}$} \\
\hline Total & 64.71 & 57.75 & 71.67 \\
Post & 72.71 & 63.30 & 82.12 \\
Pre & 61.75 & 52.97 & 70.53 \\
\hline
\end{tabular}

FIGURE 3 | Time-dependent AUC at 10 years post-surgery with 95\% Cl.
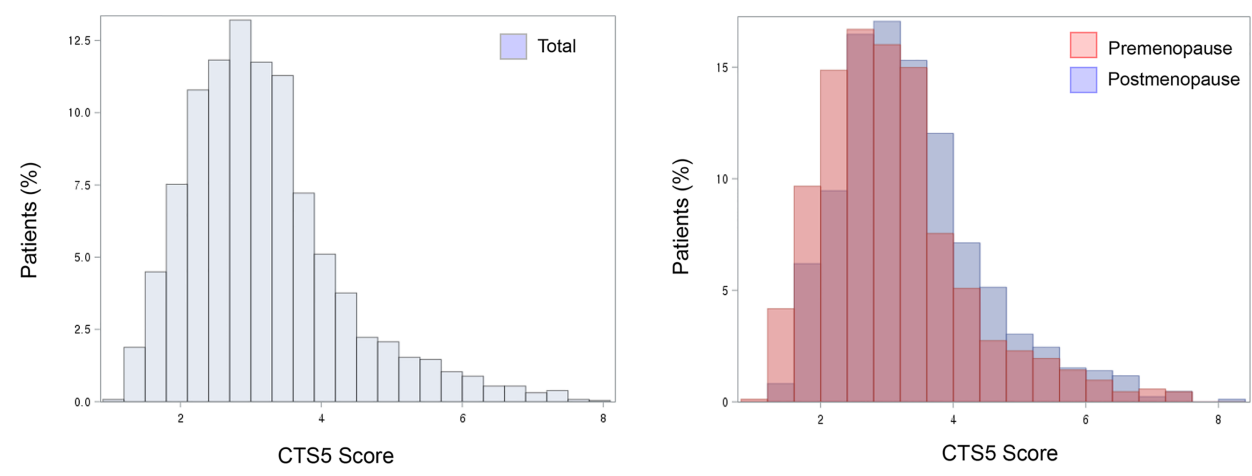

FIGURE 4 | Histogram of CTS5 scores.

groups by creating new cutoff values suitable for Korean patients. Accordingly, we plan to create a web-based search tool suitable for use in Korea.

When the CTS5 calculator was developed, HER2-positive patients were included despite the small population. Because the diagnostic technique has not been applied, such as silverenhanced in situ hybridization in our data prior to 2003, it is not clear to describe the HER2 status. Due to development of HER2 gene amplification diagnosis, HER2 status can be accurately described, allowing not only chemotherapy, but targeted therapy to be administered. HER2-positive breast cancer is different from hormone receptor-positive breast cancer in terms of molecular biology, and further study is needed to predict late DR excluding the HER2-positive subtype. In addition, this study did not confirm the difference in CTS5 risk among premenopausal women according to use of $\mathrm{GnRH}$ agonists because of the small population (156 patients) of premenopausal patients received GnRH agonists. GnRH agonists have been used to suppress ovarian function in young patients with luminal-type breast cancer and premenopausal patients with high risk for DR due to poor prognosis $(26,27)$. In the SOFT-TEXT and ASTRRA trials, it was found that the prognosis of young breast cancer patients could be improved depending upon the use of GnRH agonists (28-31). In the future, additional CTS5 evaluation is required according to the use of $\mathrm{GnRH}$ agonists in premenopausal women, and it is necessary to evaluate the late prediction rate according to the combination of CTS5 and multigene assay by GnRH agonist use.

CTS5 can be prognostic, but risk evaluation is dependent upon traditional clinicopathologic parameters such as tumor size, tumor grade, and nodal status; it cannot provide customized guidance for each individual. Therefore, its combination with a multigene assay is important for deciding whether to extend endocrine therapy $(17,32)$. Furthermore, in addition to a clinical calculator, such as CTS5, and a multigene assay, further combination with an immunohistochemistry assay and radiographic imaging (computed tomography, positronemission tomography, and magnetic resonance imaging) or tumor marker assessments is important for predicting patient prognosis $(25,33)$. 
In conclusion, although CTS5 was created to support decision-making by clinicians about extending adjuvant endocrine therapy in postmenopausal women with positive hormonal receptors, there are limitations in predicting late DR in premenopausal women. For populations of premenopausal women with greater rates of breast cancer, a modified scoring system for late DR prediction is needed so as not to underestimate the recurrence risk.

\section{DATA AVAILABILITY STATEMENT}

The original contributions presented in the study are included in the article/Supplementary Materials, further inquiries can be directed to the corresponding author.

\section{ETHICS STATEMENT}

The present study was approved by the Review Committees (no. 2020-09-143). The patients/participants provided their written informed consent to participate in this study.

\section{REFERENCES}

1. Pan H, Gray R, Braybrooke J, Davies C, Taylor C, McGale P, et al. 20-Year Risks of Breast-Cancer Recurrence After Stopping Endocrine Therapy at 5 Years. N Engl J Med (2017) 377(19):1836-46. doi: 10.1056/NEJMoa1701830

2. Burstein HJ, Temin S, Anderson H, Buchholz TA, Davidson NE, Gelmon KE, et al. Adjuvant Endocrine Therapy for Women With Hormone ReceptorPositive Breast Cancer: American Society of Clinical Oncology Clinical Practice Guideline Focused Update. J Clin Oncol (2014) 32(21):2255-69. doi: 10.1200/JCO.2013.54.2258

3. Early Breast Cancer Trialists' Collaborative Group. Effects of Chemotherapy and Hormonal Therapy for Early Breast Cancer on Recurrence and 15-Year Survival: An Overview of the Randomised Trials. Lancet (2005) 365 (9472):1687-717. doi: 10.1016/S0140-6736(05)66544-0

4. Fisher B, Costantino JP, Wickerham DL, Cecchini RS, Cronin WM, Robidoux A, et al. Tamoxifen for the Prevention of Breast Cancer: Current Status of the National Surgical Adjuvant Breast and Bowel Project P-1 Study. J Natl Cancer Inst (2005) 97(22):1652-62. doi: 10.1093/jnci/dji372

5. Winer EP, Hudis C, Burstein HJ, Wolff AC, Pritchard KI, Ingle JN, et al. American Society of Clinical Oncology Technology Assessment on the Use of Aromatase Inhibitors as Adjuvant Therapy for Postmenopausal Women With Hormone Receptor-Positive Breast Cancer: Status Report 2004. J Clin Oncol (2005) 23(3):619-29. doi: 10.1200/JCO.2005.09.121

6. Colleoni M, Giobbie-Hurder A. Benefits and Adverse Effects of Endocrine Therapy. Ann Oncol (2010) 21 Suppl 7:vii107-11. doi: 10.1093/annonc/ mdq281

7. Li L, Chang B, Jiang X, Fan X, Li Y, Li T, et al. Clinical Outcomes Comparison of 10 Years Versus 5 Years of Adjuvant Endocrine Therapy in Patients With Early Breast Cancer. BMC Cancer (2018) 18(1):977. doi: 10.1186/s12885-0184878-4

8. Davies C, Pan H, Godwin J, Gray R, Arriagada R, Raina V, et al. Long-Term Effects of Continuing Adjuvant Tamoxifen to 10 Years Versus Stopping at 5 Years After Diagnosis of Oestrogen Receptor-Positive Breast Cancer: ATLAS, a Randomised Trial. Lancet (2013) 381(9869):805-16. doi: 10.1016/S01406736(12)61963-1

9. Dowsett M, Sestak I, Regan MM, Dodson A, Viale G, Thurlimann B, et al. Integration of Clinical Variables for the Prediction of Late Distant Recurrence in Patients With Estrogen Receptor-Positive Breast Cancer Treated With 5

\section{AUTHOR CONTRIBUTIONS}

J-HL performed literature search, the data analysis, and drafted the manuscript. SL and BC performed revised the manuscript. JY performed literature investigation and reviewing. JEL SK, and SN performed supervision and reviewing. JR designed the concept of article. All authors contributed to the article and approved the submitted version.

\section{FUNDING}

This study was supported by the Korea Breast Cancer Foundation (no. PHO020413) and the biostatistics team of the Statistics and Data Center, Research Institute for Future Medicine, Samsung Medical Center.

\section{SUPPLEMENTARY MATERIAL}

The Supplementary Material for this article can be found online at: https://www.frontiersin.org/articles/10.3389/fonc.2021. 691277/full\#supplementary-material

Years of Endocrine Therapy: Cts5. J Clin Oncol (2018) 36(19):1941-8. doi: 10.1200/JCO.2017.76.4258

10. Sestak I, Dowsett M, Zabaglo L, Lopez-Knowles E, Ferree S, Cowens JW, et al. Factors Predicting Late Recurrence for Estrogen Receptor-Positive Breast Cancer. J Natl Cancer Inst (2013) 105(19):1504-11. doi: 10.1093/jnci/djt244

11. Arimidex T, Forbes JF, Cuzick J, Buzdar A, Howell A, Tobias JS, et al. Effect of Anastrozole and Tamoxifen as Adjuvant Treatment for Early-Stage Breast Cancer: 100-Month Analysis of the ATAC Trial. Lancet Oncol (2008) 9(1):4553. doi: 10.1016/S1470-2045(07)70385-6

12. Cuzick J, Sestak I, Baum M, Buzdar A, Howell A, Dowsett M, et al. Effect of Anastrozole and Tamoxifen as Adjuvant Treatment for Early-Stage Breast Cancer: 10-Year Analysis of the ATAC Trial. Lancet Oncol (2010) 11 (12):1135-41. doi: 10.1016/S1470-2045(10)70257-6

13. Sung H, Rosenberg PS, Chen WQ, Hartman M, Lim WY, Chia KS, et al. Female Breast Cancer Incidence Among Asian and Western Populations: More Similar Than Expected. J Natl Cancer Inst (2015) 107(7):1-7. doi: 10.1093/jnci/djv107

14. Breast International Group 1-98 Collaborative Group, Thurlimann B, Keshaviah A, Coates AS, Mouridsen H, Mauriac L, et al. A Comparison of Letrozole and Tamoxifen in Postmenopausal Women With Early Breast Cancer. N Engl J Med (2005) 353(26):2747-57. doi: 10.1056/NEJMoa052258

15. Regan MM, Neven P, Giobbie-Hurder A, Goldhirsch A, Ejlertsen B, Mauriac L, et al. Assessment of Letrozole and Tamoxifen Alone and in Sequence for Postmenopausal Women With Steroid Hormone Receptor-Positive Breast Cancer: The BIG 1-98 Randomised Clinical Trial at 8.1 Years Median FollowUp. Lancet Oncol (2011) 12(12):1101-8. doi: 10.1016/S1470-2045(11)70270-4

16. Blanche P, Dartigues J-F, Jacqmin-Gadda H. Estimating and Comparing Time-Dependent Areas Under Receiver Operating Characteristic Curves for Censored Event Times With Competing Risks. Stat Med (2013) 32:5381-97. doi: $10.1002 / \operatorname{sim} .5958$

17. Sestak I, Buus R, Cuzick J, Dubsky P, Kronenwett R, Denkert C, et al. Comparison of the Performance of 6 Prognostic Signatures for Estrogen Receptor-Positive Breast Cancer: A Secondary Analysis of a Randomized Clinical Trial. JAMA Oncol (2018) 4(4):545-53. doi: 10.1001/jamaoncol. 2017.5524

18. Yoo KY, Kang D, Park SK, Kim SU, Kim SU, Shin A, et al. Epidemiology of Breast Cancer in Korea: Occurrence, High-Risk Groups, and Prevention. J Korean Med Sci (2002) 17(1):1-6. doi: 10.3346/jkms.2002.17.1.1 
19. Villasco A, Agnelli F, D'Alonzo M, Accomasso F, Sismondi P, Biglia N. Validation of CTS5 on a Retrospective Cohort of Real-Life Pre- and Postmenopausal Patients Diagnosed With Estrogen Receptor-Positive Breast Cancers: Is it Prognostic? Clin Breast Cancer (2021) 21(1):e53-62. doi: 10.1016/j.clbc.2020.06.008

20. Lee J, Cha C, Ahn SG, Kim D, Park S, Bae SJ, et al. Validation of Clinical Treatment Score Post-5 Years (CTS5) Risk Stratification in Premenopausal Breast Cancer Patients and Ki-67 Labelling Index. Sci Rep (2020) 10(1):16850. doi: 10.1038/s41598-020-74055-3

21. Arpino G, Wiechmann L, Osborne CK, Schiff R. Crosstalk Between the Estrogen Receptor and the HER Tyrosine Kinase Receptor Family: Molecular Mechanism and Clinical Implications for Endocrine Therapy Resistance. Endocr Rev (2008) 29(2):217-33. doi: 10.1210/er.2006-0045

22. Wang C, Chen C, Lin Y, Zhou Y, Mao F, Zhu H, et al. Validation of CTS5 Model in Large-Scale Breast Cancer Population and the Impact of Menopausal and HER2 Status on its Prognostic Value. Sci Rep (2020) 10 (1):4660. doi: 10.1038/s41598-020-61648-1

23. Noordhoek I, Blok EJ, Meershoek-Klein Kranenbarg E, Putter H, Duijm-de Carpentier M, Rutgers EJT, et al. Overestimation of Late Distant Recurrences in High-Risk Patients With Er-Positive Breast Cancer: Validity and Accuracy of the CTS5 Risk Score in the TEAM and IDEAL Trials. J Clin Oncol (2020) 38 (28):3273-81. doi: 10.1200/JCO.19.02427

24. Kwon MJ, Lee JE, Jeong J, Woo SU, Han J, Kang BI, et al. Comparison of GenesWell Bct Score With Oncotype Dx Recurrence Score for Risk Classification in Asian Women With Hormone Receptor-Positive, Her2Negative Early Breast Cancer. Front Oncol (2019) 9:667. doi: 10.3389/ fonc. 2019.00667

25. Dowling RJO, Kalinsky K, Hayes DF, Bidard FC, Cescon DW, Chandarlapaty S, et al. Toronto Workshop on Late Recurrence in Estrogen Receptor-Positive Breast Cancer: Part 1: Late Recurrence: Current Understanding, Clinical Considerations. JNCI Cancer Spectr (2019) 3(4):pkz050. doi: 10.1093/jncics/pkz050

26. Huerta-Reyes M, Maya-Nunez G, Perez-Solis MA, Lopez-Munoz E, Guillen N, Olivo-Marin JC, et al. Treatment of Breast Cancer With GonadotropinReleasing Hormone Analogs. Front Oncol (2019) 9:943. doi: 10.3389/ fonc. 2019.00943

27. Jonat W, Kaufmann M, Blamey RW, Howell A, Collins JP, Coates A, et al. A Randomised Study to Compare the Effect of the Luteinising Hormone Releasing Hormone (LHRH) Analogue Goserelin With or Without Tamoxifen in Pre- and
Perimenopausal Patients With Advanced Breast Cancer. Eur J Cancer (1995) 31a (2):137-42. doi: 10.1016/0959-8049(94)00415-2

28. Francis PA, Pagani O, Fleming GF, Walley BA, Colleoni M, Lang I, et al. Tailoring Adjuvant Endocrine Therapy for Premenopausal Breast Cancer. N Engl J Med (2018) 379(2):122-37. doi: 10.1056/NEJMoa1803164

29. Noh WC, Lee JW, Nam SJ, Park S, Im SA, Lee ES, et al. Role of Adding Ovarian Function Suppression to Tamoxifen in Young Women With Hormone-Sensitive Breast Cancer Who Remain Premenopausal or Resume Menstruation After Chemotherapy: The ASTRRA Study. J Clin Oncol (2018) 36(15 Suppl):502. doi: 10.1200/JCO.2018.36.15_suppl.502

30. Francis PA, Regan MM, Fleming GF, Lang I, Ciruelos E, Bellet M, et al. Adjuvant Ovarian Suppression in Premenopausal Breast Cancer. N Engl J Med (2015) 372(5):436-46. doi: 10.1056/NEJMoa1412379

31. LHRH-agonists in Early Breast Cancer Overview group, Cuzick J, Ambroisine L, Davidson N, Jakesz R, Kaufmann M, et al. Use of Luteinising-HormoneReleasing Hormone Agonists as Adjuvant Treatment in Premenopausal Patients With Hormone-Receptor-Positive Breast Cancer: A Meta-Analysis of Individual Patient Data From Randomised Adjuvant Trials. Lancet (2007) 369(9574):1711-23. doi: 10.1016/S0140-6736(07)60778-8

32. Paik S, Shak S, Tang G, Kim C, Baker J, Cronin M, et al. A Multigene Assay to Predict Recurrence of Tamoxifen-Treated, Node-Negative Breast Cancer. N Engl J Med (2004) 351(27):2817-26. doi: 10.1056/NEJMoa041588

33. Harris LN, Ismaila N, McShane LM, Andre F, Collyar DE, Gonzalez-Angulo $\mathrm{AM}$, et al. Use of Biomarkers to Guide Decisions on Adjuvant Systemic Therapy for Women With Early-Stage Invasive Breast Cancer: American Society of Clinical Oncology Clinical Practice Guideline. J Clin Oncol (2016) 34(10):1134-50. doi: 10.1200/JCO.2015.65.2289

Conflict of Interest: The authors declare that the research was conducted in the absence of any commercial or financial relationships that could be construed as a potential conflict of interest.

Copyright (c) 2021 Lee, Lee, Chae, Yu, Lee, Kim, Nam and Ryu. This is an open-access article distributed under the terms of the Creative Commons Attribution License (CC BY). The use, distribution or reproduction in other forums is permitted, provided the original author(s) and the copyright owner(s) are credited and that the original publication in this journal is cited, in accordance with accepted academic practice. No use, distribution or reproduction is permitted which does not comply with these terms. 